Bull. Austral. Math. Soc.

$57 \mathrm{M} 05,20 \mathrm{E} 26,57 \mathrm{M} 50,57 \mathrm{~N} 35$

VoL. 55 (1997) [261-279]

\title{
A 3-MANIFOLD WITH A NON-SUBGROUP-SEPARABLE FUNDAMENTAL GROUP
}

\section{Saburo Matsumoto}

\begin{abstract}
We examine a 3-manifold $\Gamma$ whose fundamental group is known to be nonsubgroup-separable (non-LERF). We show that this manifold $\Gamma$ is a graph manifold and that the subgroup known to be non-separable is not geometric. On the other hand, there are incompressible surfaces immersed in the manifold which do not lift to embeddings in any finite-degree covering space. We then prove that these bad incompressible surfaces must have non-empty boundary.
\end{abstract}

\section{Preliminaries}

A group $G$ is said to be residually finite (RF) if, for any $\gamma \in G \backslash\{1\}$, there exists a finite-index subgroup $G_{1}$ not containing $\gamma$. If $S$ is a subgroup of $G$, then $G$ is said to be $S$-residually finite $(S-R F)$ if, for any $\gamma \in G \backslash S$, there exists a finite-index subgroup $G_{1}$ of $G$ containing $S$ but not $\gamma$. We say that $G_{1}$ separates $S$ from $\gamma$ and that $S$ is a separable subgroup of $G$. $G$ is called locally extended residually finite ( $L E R F)$ if $G$ is $S$-RF for every finitely generated subgroup $S$ of $G$. LERF groups are sometimes referred to as subgroup separable groups. In this paper, the terms "LERF" and "subgroup separable" are used interchangeably.

If $G$ is LERF, then so are any subgroups of $G$ (this is obvious) and any finite extensions of $G$ (Scott [13]). See Allenby and Gregorac [1], Magnus [11], and Scott [13] for various nice properties of these groups.

The following lemma, a direct corollary to a result in [13], provides an essential link between group theory and geometric topology.

Lemma 1.1. Let $M$ be a topological 3-manifold with $\pi_{1}(M)=G$. If $G$ is $L E R F$, then any incompressible surface $F$ immersed in $M$ by $f: F \rightarrow M$ can be lifted to an embedding $f_{1}: F \rightarrow \widehat{M}$, where $\widehat{M}$ is a finite-degree covering space for $M$.

REMARK. By incompressible surface, we mean that the map $f$ induces a $\pi_{1}$-injective $\operatorname{map} f_{*}: F \rightarrow M$.

We say that a compact irreducible 3-manifold $M$ is a graph manifold if each component of $M \backslash \mathcal{T}$ is a Seifert fibre space, where $\mathcal{T}$ is the family of tori in the canonical torus

Received 9th April, 1996

Research partially supported by Australian Research Council.

Copyright Clearance Centre, Inc. Serial-fee code: 0004-9729/97 \$A2.00+0.00. 
decomposition. Each Seifert fibre piece is often called a vertex manifold. In this class we include those manifolds that are obtained by gluing a pair (or pairs) of boundary torus components of one single Seifert fibre space.

In 1978, Scott showed that all surface groups, all Fuchsian groups, and the fundamental groups of Seifert fibre spaces are LERF [13] (also, see Hempel [7], Hall [5], Burns [2], and Karrass and Burns [8]). This, of course, implies that any circle bundle over a surface has a LERF fundamental group. However, Scott wrote, "I am unable to decide whether the same holds for bundles over $S^{1}$ with fibre a surface. It seems quite possible that this is false" (p.565).

As Scott speculated, almost ten years later, Burns, Karrass, and Solitar [3] found a non-LERF group which is the fundamental group of a surface bundle over $S^{1}$. This resulted in the discovery of more 3-manifolds with non-LERF fundamental groups (see Long and Niblo [9], for example). In this paper we shall study the example given in [3].

\section{NON-LERF GROUP $K$}

We begin by presenting the group $K$ proved non-LERF in [3]. Their paper specifies a finitely generated subgroup $H$ of $K$ and an element $\gamma \in K \backslash H$ which cannot be separated from $H$ by a finite-index subgroup of $K$ containing $H$. In their presentation,

and

$$
\begin{aligned}
& K=\left\langle y, \alpha, \beta \mid y^{-1} \alpha y=\alpha \beta,: y^{-1} \beta y=\beta\right\rangle, \\
& H=\left\langle\alpha^{-1}, y \alpha^{-1} y^{-2} \alpha\right\rangle=\left\langle\alpha, y \alpha^{-1} y^{-2}\right\rangle,
\end{aligned}
$$

$$
\gamma=\left[y, \alpha^{-2} y \alpha^{2}\right] \text {. }
$$

Here, $\left[x_{1}, x_{2}\right]$ denotes the commutator $x_{1}^{-1} x_{2}^{-1} x_{1} x_{2}$. For details of their proof, see [3]. Note that, since $\beta=\alpha^{-1} y^{-1} \alpha y$, the group $K$ can be presented by 2 elements and 1 relation instead.

This particular presentation of $K$ suggests a non-closed 3-manifold $\Gamma$ with $\pi_{1}(\Gamma)=$ $K$, obtained as follows:

Let $F$ be the punctured torus, and let $\pi_{1}(F)=\langle\alpha, \beta\rangle$, where $\alpha$ represents the longitude and $\beta$ represents the meridian of $F$. Consider $F \times I$. Identify $F \times\{0\}$ and $F \times\{1\}$ with the homeomorphism of $F$ prescribed by the two relations

$$
y^{-1} \alpha y=\alpha \beta \quad \text { and } \quad y^{-1} \beta y=\beta .
$$

The construction is shown in Figure 1. The gluing homeomorphism determined by these relations is a Dehn twist along the meridian curve $\beta$ :

$$
\alpha \longmapsto \alpha \beta, \quad \beta \longmapsto \beta .
$$




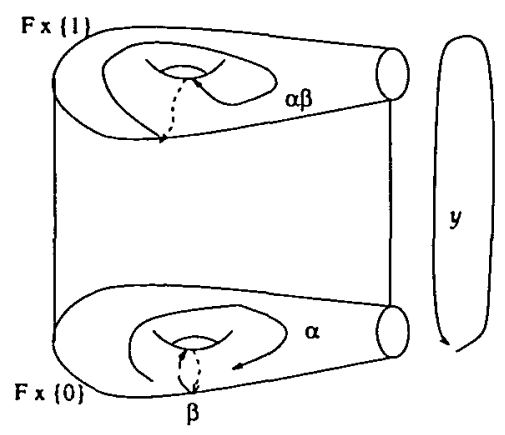

Figure 1. Non-LERF 3-manifold $\Gamma$

The resulting $\Gamma$ is simply a surface bundle over $S^{1}$. Note that $\partial \Gamma$ is the torus, and if this is "capped off" in the obvious manner, we get a closed manifold $\Gamma^{\prime}$ having the Nil geometry [14].

The following lemma is useful in analysing $\Gamma$.

LemMa 2.1. $\Gamma$ is a graph manifold consisting of one Seifert fibre space with three boundary components, two of which are identified.

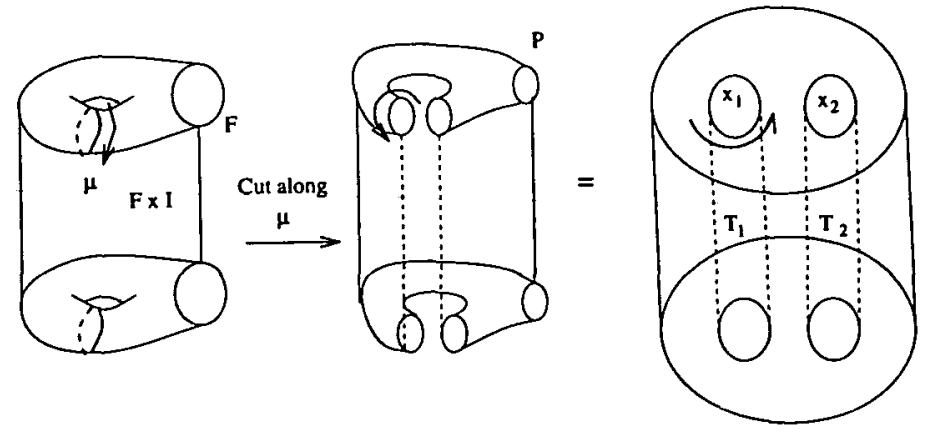

Figure 2. $\Gamma$ as a graph manifold

Proof: Let $F$ denote the punctured torus. The description above for $\Gamma$ shows that $\Gamma$ is obtained from $F \times I$ by the Dehn twist along the meridian, defined by the matrix

$$
\left(\begin{array}{ll}
1 & 0 \\
1 & 1
\end{array}\right)
$$

Now, one can cut $F$ along the meridian curve $\mu$ and get the "pair of pants" $S^{2}-3 D^{2}$, which we denote by $P$. Consider $P \times S^{1}$, the solid torus with the interior of two disjoint 
solid tori $T_{1}$ and $T_{2}$ removed (Figure 2). For $i=1,2$, let

$$
\pi_{1}\left(T_{i}\right)=\left\langle x_{i}\right\rangle \oplus\left\langle t_{i}\right\rangle \cong \mathbb{Z} \oplus \mathbb{Z}
$$

where $x_{i}$ is the meridian (horizontal in $P \times S^{1}$ ) and the $t_{i}$ is the longitude (vertical in $P \times S^{1}$ ). We shall identify $T_{1}$ and $T_{2}$ in such a way that the resulting manifold is homeomorphic to $\Gamma$. As one can see in Figure 2, the Dehn twist performed to make $\Gamma$ corresponds to "turning" $x_{1}$ one complete rotation and gluing the two tori together. In other words, by identifying $T_{1}$ and $T_{2}$ by the homeomorphism $h: T_{1} \rightarrow T_{2}$ inducing

$$
\begin{aligned}
h_{*}: \pi_{1}\left(T_{1}\right) & \rightarrow \pi_{1}\left(T_{2}\right) \quad \text { defined by } \\
x_{1} & \longmapsto x_{2} \\
t_{1} & \longmapsto x_{2}+t_{2},
\end{aligned}
$$

one gets a manifold homeomorphic to $\Gamma$. The space $P \times S^{1}$ is clearly a Seifert fibre space, so $\Gamma$ is a graph manifold.

We now give yet another presentation of $K$.

LEммA 2.2. The presentation

$$
K=\left\langle y, \alpha, \beta \mid y^{-1} \alpha y=\alpha \beta, y^{-1} \beta y=\beta\right\rangle,
$$

is Tietze equivalent to (and thus isomorphic to) the presentation

$$
\left\langle y, y_{1}, \alpha \mid \alpha^{-1} y \alpha=y_{1},\left[y, y_{1}\right]=1\right\rangle .
$$

Proof: Simply substitute $\beta=y y_{1}^{-1}$, and verify that

$$
\begin{aligned}
K & =\left\langle y, \alpha, \beta \mid y^{-1} \alpha y=\alpha \beta,[\beta, y]=1\right\rangle \\
& =\left\langle y, \alpha, y y_{1}^{-1} \mid y^{-1} \alpha y=\alpha y y_{1}^{-1},\left[y y_{1}^{-1}, y\right]=1\right\rangle \\
& =\left\langle y, \alpha, y_{1} \mid y^{-1} \alpha y=\alpha y y_{1}^{-1},\left[y, y_{1}\right]=1\right\rangle \\
& =\left\langle y, y_{1}, \alpha \mid y^{-1} \alpha y=\alpha y_{1}^{-1} y,\left[y, y_{1}\right]=1\right\rangle \\
& =\left\langle y, y_{1}, \alpha \mid \alpha^{-1} y^{-1} \alpha=y_{1}^{-1},\left[y, y_{1}\right]=1\right\rangle \\
& =\left\langle y, y_{1}, \alpha \mid \alpha^{-1} y \alpha=y_{1},\left[y, y_{1}\right]=1\right\rangle
\end{aligned}
$$

is indeed a sequence of Tietze transformations (and obvious substitutions and manipulations).

With this new presentation of $K$, we can now realise $\Gamma$ in yet another way. Since $y$ and $y_{1}$ commute in $G$, let $T$ be the torus whose meridian curve is represented by $y$ and longitude curve by $y_{1}$. Start with $T \times I$, and take the regular (2-dimensional) 


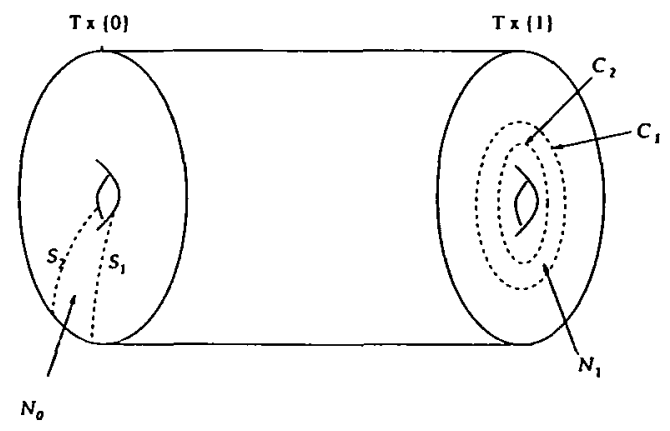

Figure 3. The two annuli to be identified

neighbourhood $N_{0}$ of the meridian curve on $T \times\{0\}$ (see Figure 3). $\partial N_{0}$ is two circles $S_{1}$ and $S_{2}$. Similarly, take the neighbourhood $N_{1}$ of the longitude curve of $T \times\{1\}$. Refer to the two boundary circles of $\partial N_{1}$ as $C_{1}$ and $C_{2}$.

Now take an annulus $A$ with $\partial A=\partial_{1} \cup \partial_{2}$, and construct $A \times I$. Identify $A \times\{0\}$ with $N_{0}$ and $A \times\{1\}$ with $N_{1}$ such that for $i=1,2$,

$$
A_{i}=\partial_{i} \times I \text { joins } S_{i} \text { with } C_{i} .
$$

Since the $\partial_{i}$ are circles, the $A_{i}$ are also annuli. We shall refer to these as connecting annuli (See Figure 4).

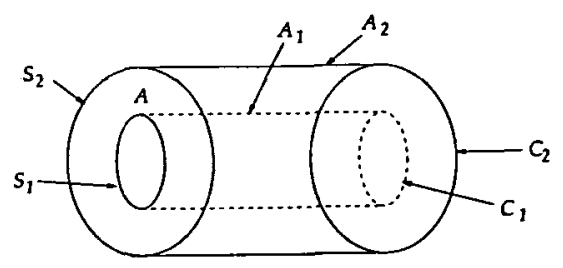

Figure 4. The connecting annuli $A_{1}, A_{2}$

The resulting manifold $M$ is a 3-manifold with boundary, and $\pi_{1}(M)=G$, where $y, y_{1} \in \pi_{1}(T)$, and $\alpha$ is a primitive loop going through the attached annuli, carrying $y$ to $y_{1}$. Note that we may have actually constructed two distinct manifolds here, depending on how we labelled the $S_{i}$ and the $C_{i}$. Indeed, Theorem 2.3 below shows that two distinct manifolds can be constructed this way and that one of them is homeomorphic to $\Gamma$. Here, we are assuming that the circles $S_{i}$ are oriented in the same way, and so are the $C_{i}$. Observe that, in either way,

$$
\partial M=\left((T \times\{0\}) \backslash N_{0}\right) \cup A_{1} \cup\left((T \times\{1\}) \backslash N_{1}\right) \cup A_{2},
$$


where $A_{i}$ is the annulus connecting $S_{i}$ to $C_{i}$. This is a union of four annuli making up a single torus boundary component.

THEOREM 2.3. The above construction gives two distinct 3-manifolds, $M_{1}$ and $M_{2}$, and one of them is homeomorphic to $\Gamma$.

Proof: After fixing the $C_{i}$ and the $S_{i}$, one gluing (taking $C_{1}$ to $S_{1}$ ) gives an orientable manifold while the other gluing gives a non-orientable one, so it is clear that we get two distinct manifolds. Let $M_{1}$ be the manifold constructed with the labeling given in Figure 5, and the annuli join $S_{1}$ with $C_{1}$ and $S_{2}$ with $C_{2}$. Pick a base point of $M_{1}$ as shown in Figure 5, and orient each generator curve as indicated by the arrows. We see that

$$
\pi_{1}\left(M_{1}\right)=\left\langle\alpha, y, y_{1} \mid\left[y, y_{1}\right]=1, \alpha^{-1} y \alpha=y_{1}\right\rangle \cong K,
$$

as required. Consider now $\partial \Gamma \cong T^{2} . \pi_{1}(\partial \Gamma)$ is generated by $y$ and $\alpha \beta \alpha^{-1} \beta^{-1}$. On the other hand, as stated before, $\partial M_{1}$ is also homeomorphic to the torus, diagrammatically shown in Figure 6. $\pi_{1}\left(\partial M_{1}\right)$ is thus generated by $y$ and $\alpha y \alpha^{-1} y_{1}$.

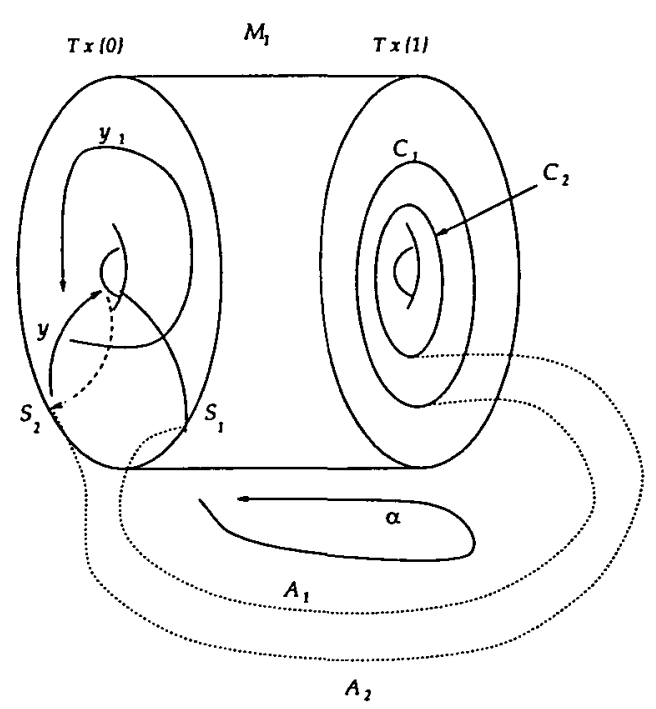

Figure 5. $\Gamma$ and $\pi_{1}(\Gamma)$ as an HNN extension 
Using the identity $\beta=\alpha^{-1} y^{-1} \alpha y$ in $K$, we get

$$
\begin{aligned}
\pi_{1}(\partial \Gamma) & =\langle y\rangle \oplus\left\langle\alpha \beta \alpha^{-1} \beta^{-1}\right\rangle \\
& =\langle y\rangle \oplus\left\langle\alpha \alpha^{-1} y^{-1} \alpha y \alpha^{-1} y^{-1} \alpha^{-1} y \alpha\right\rangle \\
& =\langle y\rangle \oplus\left\langle\alpha y \alpha^{-1} y^{-1} \alpha^{-1} y \alpha\right\rangle \\
& =\langle y\rangle \oplus\left\langle\alpha y \alpha^{-1} y^{-1} y_{1}\right\rangle \\
& =\langle y\rangle \oplus\left\langle\alpha y \alpha^{-1} y_{1} y^{-1}\right\rangle \quad \text { since } y \text { and } y_{1} \text { commute } \\
& =\langle y\rangle \oplus\left\langle\alpha y \alpha^{-1} y_{1}\right\rangle \\
& =\pi_{1}\left(\partial M_{1}\right)
\end{aligned}
$$

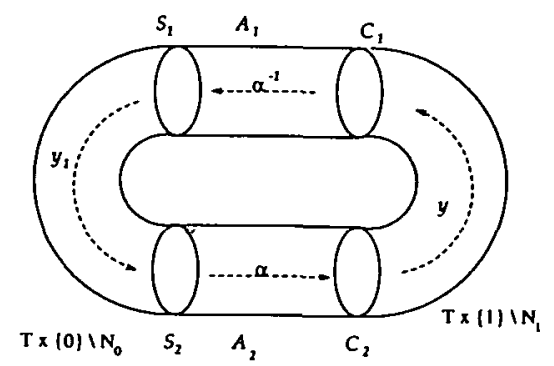

Figure 6 . The boundary $\partial \Gamma$

Hence, we have a group isomorphism $\pi_{1}(\Gamma) \rightarrow \pi_{1}\left(M_{1}\right)$ such that it maps $\pi_{1}(\partial \Gamma)$ isomorphically onto $\pi_{1}\left(\partial M_{1}\right)$. By Waldhausen's Theorem, we conclude that $\Gamma \cong M_{1}$.

\section{The NON-SEPARABLE SUBGROUP OF $K$}

As mentioned in Section 1, one of the implications of $\pi_{1}(M)$ being non-LERF is that there may be an incompressible surface $S$ (immersed in $M$ ) not lifting to an embedding in any finite cover of $M$. If so, $\pi_{1}(S) \subset \pi_{1}(M)$ is non-separable. Thus, if $\Gamma$ does contain such a surface $S$, then $H$ is a candidate for $\pi_{1}(S)$. We shall see later that this is not the case (see Theorem 3.4), but before proving it, we must examine $H$ further. The key machinery used here is the following, due to Britton [10].

Lemma 3.1. Suppose $G$ is a group with subgroups $A$ and $B, \eta: A \rightarrow B$ is an isomorphism, and $\widehat{G}$ is an HNN extension of $G$ defined as

$$
\widehat{G}=\left\langle G, t \mid t^{-1} a t=\eta(a), a \in A\right\rangle .
$$


Let $g_{i} \in G$ and $\varepsilon_{i}= \pm 1$, and suppose the word

$$
w=g_{0} t^{e_{1}} g_{1} \ldots t^{e_{n}} g_{n}
$$

(where $n \geqslant 1$ ) does not contain any subword $t^{-1} g_{i} t, g_{i} \in A$, or $t g_{j} t^{-1}, g_{j} \in B$. Then $w$ is not the identity in $\widehat{G}$.

An immediate corollary is the following:

Corollary 3.2. Let $G$ and $\widehat{G}$ be defined as above, and let $g \in G$ be an element such that $g^{k} \notin(A \cup B)$ for all $k \neq 0$. Then, the subgroup $\langle g, t\rangle$ of $\widehat{G}$ is free of rank 2.

Proof: We need to show that the homomorphism

$$
\Phi:\langle g, t\rangle \text { (as a free group) } \rightarrow\langle g, t\rangle \text { (as a subgroup of } \widehat{G} \text { ) }
$$

is injective. So let $w$ be a word in the free group $\langle g, t\rangle$, reduced in the "free-group sense," and suppose $\Phi(w)=1$ in $\widehat{G}$. Clearly $w \neq g^{k}(k \neq 0)$, since $g^{k} \notin A \cup B$. Hence, $w=1$ already, or $w$ contains at least one $t$, so we can apply Britton's Lemma 3.1 (as $n \geqslant 1)$ and conclude that $w$ contains a subword $t^{-1} g_{i} t$ or $t g_{j}^{-1} t^{-1}\left(g_{i} \in A\right.$ or $\left.g_{j} \in B\right)$. Now $g_{i}, g_{j}$ must be $g^{k}$ for some $k \neq 0$, since $w$ is a reduced word in $g$ and $t$. But $g^{k} \in A$ or $B$ is contrary to our hypothesis. Hence, $w=1$, and $\Phi$ is injective.

Corollary 3.3. $H$ is isomorphic to the free group of rank 2.

Proof: Using the identity $y_{1}=\alpha^{-1} y \alpha$, we see that the subgroup $H$ is generated by $\alpha$ and $y \alpha^{-1} y^{-2} \alpha=y\left(\alpha^{-1} y \alpha\right)^{-2}=y y_{1}^{-2}$.

$K$ is an HNN extension of the torus group generated by $y$ and $y_{1}$. Let $G=$ $\langle y\rangle \oplus\left\langle y_{1}\right\rangle$, and let $A=\langle y\rangle, B=\left\langle y_{1}\right\rangle$; define $\eta(y)=\alpha^{-1} y \alpha=y_{1}$. Then, $K=\widehat{G}$ in the notation of Lemma 3.1, and we can apply Corollary 3.2 since there is no $k \neq 0$ such that $\left(y y_{1}^{-2}\right)^{k} \in A$ or $B$ in $G$. Hence, $H=\left\langle y y_{1}^{-2}, \alpha\right\rangle$ is free of rank 2 .

Now, the classification result for compact connected surfaces gives all the surfaces $S$ such that $\pi_{1}(S) \cong F_{2}$, the free group of rank $2 . \partial S \neq \varnothing$, so we get

$$
H_{0}(S)=\mathbb{Z}, H_{1}(S)=\mathbb{Z} \oplus \mathbb{Z}, \text { and } H_{2}(S)=0,
$$

and thus $\chi(S)=-1$. Hence, $S$ is homeomorphic to the pair of pants, the punctured torus, the punctured Moebius band, or the punctured Klein bottle.

Now, we are ready to prove the main result of this section.

TheOREM 3.4. Let $\Gamma$ be the manifold described in Section 2, and present $\pi_{1}(\Gamma)=K$ as above. Suppose $f: S \rightarrow \Gamma$ is a proper two-sided immersion of a compact connected surface such that $f_{*}: \pi_{1}(S) \rightarrow \pi_{1}(\Gamma)$ is an injection. Then, $f_{*}\left(\pi_{1}(S)\right)$ cannot be $H$. 
REMARK. By proper immersion, we mean that $f$ is a local embedding such that $f^{-1}(\partial \Gamma)=\partial S$.

Proof: Of the four surfaces $S$ whose fundamental group is free of rank 2, the two non-orientable ones cannot be immersed in a two-sided way. Hence, we need to consider only the following two cases.

CASE 1. First, suppose $S$ is the pair of pants. Since $f$ is a proper map, $\partial S$, which is three disjoint circles, must be mapped into the boundary torus of $\Gamma$. Let

$$
f_{*}(a)=\bar{a}, f_{*}(b)=\bar{b},
$$

where $a, b$ are shown in Figure 7 , and $\bar{a}, \bar{b}$ are their images in $\pi_{1}(\Gamma)$. The base point of $\pi_{1}(S)$ is on the boundary component associated with $a$, so $\bar{a} \in \pi_{1}(\partial \Gamma)$. As for $\bar{b}$, since a conjugate of $b$ represents a boundary component of $S, \bar{b}$ is conjugate to $\bar{c} \in \pi_{1}(\partial \Gamma)$ by some $g \in \pi_{1}(\Gamma)$. Hence, $\bar{b}=g^{-1} \bar{c} g$. Note that $g$ may not be in $\pi_{1}(\partial \Gamma)$. However, we know that $[\bar{a}, \bar{c}]=1$ since $\bar{a}, \bar{c} \in \pi_{1}(\partial \Gamma)$, an Abelian group. Therefore, we have $f_{*}\left(\pi_{1}(S)\right)=\left\langle\bar{a}, g^{-1} \bar{c} g\right\rangle$, a subgroup of $K$.

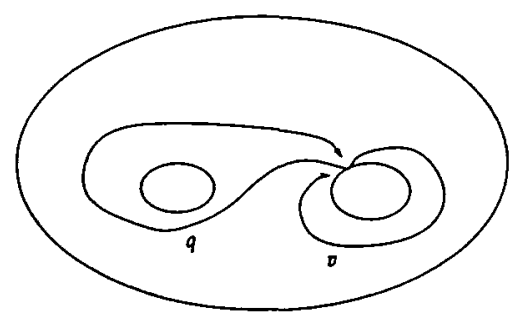

Figure 7. $S$, the "pair of pants"

Now, let $A: K \rightarrow K / K^{\prime}$ be the Abelianisation map. Then, $K / K^{\prime} \cong \mathbb{Z}^{2}$ is generated by the images of $y$ and $\alpha$, and $A(\beta)=1$. Note that

$$
A\left(\pi_{1}(\partial \Gamma)\right)=A\left(\left\langle\alpha \beta \alpha^{-1} \beta^{-1}\right\rangle \oplus\langle y\rangle\right)=\langle y\rangle \cong \mathbb{Z} .
$$

Now, consider the image of the subgroup $f_{*}\left(\pi_{1}(S)\right)$ under $A$ in $K / K^{\prime}$.

$$
A\left(f_{*}\left(\pi_{1}(S)\right)\right)=A\left(\left\langle\bar{a}, g^{-1} \bar{c} g\right\rangle\right)=A(\langle\bar{a}, \bar{c}\rangle) \subset A\left(\pi_{1}(\partial \Gamma)\right)=\langle y\rangle .
$$

On the other hand,

$$
A(H)=A\left(\left\langle\alpha, y \alpha^{-1} y^{-2}\right\rangle\right)=A(\langle\alpha, y\rangle)=\langle\alpha\rangle \oplus\langle y\rangle .
$$

Hence, $f_{*}\left(\pi_{1}(S)\right) \neq H$ as their images under $A$ are not even isomorphic. 
CASE 2. Next, suppose $S$ is the punctured torus. Let $\pi_{1}(S)=\langle a, b\rangle$, and so $\pi_{1}(\partial S)=\left\langle a b a^{-1} b^{-1}\right\rangle$. Since $\pi_{1}(\partial S)$ lies in the commutator subgroup of $\pi_{1}(S)$, its image $f_{*}\left(\pi_{1}(\partial S)\right) \subset \pi_{1}(\partial \Gamma) \cap K^{\prime}=\left\langle\alpha \beta \alpha^{-1} \beta^{-1}\right\rangle$.

Now, consider a new (closed) 3-manifold $N$ obtained by "capping off" the boundary torus of $\Gamma$ in such a way that $[\alpha, \beta]$ gets "killed" in $\pi_{1}(N)$. In other words, we attach a solid torus on $\partial \Gamma$ so that

$$
\pi_{1}(N)=\pi_{1}(\Gamma) /\langle[\alpha, \beta]=1\rangle
$$

$N$ is a torus bundle over $S^{1}$, obtained by filling in the "puncture" of the fibre of $\Gamma$. Now,

$$
\pi_{1}(N)=\left\langle y, \alpha, \beta \mid y^{-1} \alpha y=\alpha \beta, y^{-1} \beta y=\beta,[\alpha, \beta]=1\right\rangle,
$$

so $\beta$ commutes with everything. As $S$ is mapped properly to $\Gamma$, this "capping off" process will close up $f(S)$, and $\pi_{1}(S)$ will become $\pi_{1}\left(S \cup D^{2}\right)=\pi_{1}\left(T^{2}\right)$. As the result, we have the following commutative diagram:

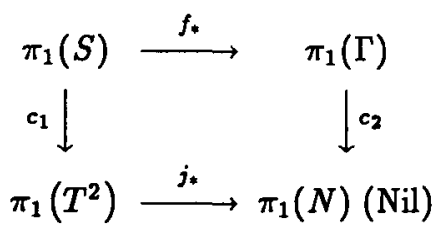

where $j_{*}$ is induced by $f_{*}$, and $c_{1}, c_{2}$ are induced by the capping operation. Note that $c_{1}, c_{2}$ are surjective. Now, $\pi_{1}\left(T^{2}\right) \cong \mathbb{Z} \oplus \mathbb{Z}$, so $j_{*} \cdot c_{1}\left(\pi_{1}(S)\right)$ is an Abelian subgroup of $\pi_{1}(N)$. Thus, $c_{2} \cdot f_{*}\left(\pi_{1}(S)\right)$ is also Abelian.

Now, suppose $H=\left\langle\alpha, y \alpha^{-1} y^{-2}\right\rangle=f_{*}\left(\pi_{1}(S)\right)$ in $\pi_{1}(\Gamma)$. Then, clearly $c_{2}(H)$ must be Abelian as well, that is, $\left[\alpha, y \alpha^{-1} y^{-2}\right]=1$ in $\pi_{1}(N)$ with the additional relation.

Observe that the question is now reduced to the algebraic problem of $H$ : whether or not $H$ becomes Abelian when the relation $[\alpha, \beta]=1$ is added. Denote $\pi_{1}(N)$ by $\bar{K}$. If $\boldsymbol{H}=f_{*}\left(\pi_{1}(S)\right)$, then $c_{2}(H)$ is Abelian, and we have

$$
\left[\alpha, y \alpha^{-1} y^{-2}\right]=\alpha^{-1} y^{2} \alpha y^{-1} \alpha y \alpha^{-1} y^{-2}=1
$$

in $\bar{K}$. Recall that in $\bar{K}, \beta$ commutes with both $\alpha$ and $y$. So we get

$$
\begin{aligned}
\beta=\beta \cdot 1 & =(\beta) \alpha^{-1} y^{2} \alpha y^{-1} \alpha y \alpha^{-1} y^{-2} \\
& =\alpha^{-1} y^{2} \alpha(\beta) y^{-1} \alpha y \alpha^{-1} y^{-2} \\
& =\alpha^{-1} y^{2} \alpha\left(\alpha^{-1} y^{-1} \alpha y\right) y^{-1} \alpha y \alpha^{-1} y^{-2} \\
& =\alpha^{-1} y \alpha^{2} y \alpha^{-1} y^{-2}
\end{aligned}
$$


Now, since $\beta^{-1}=y^{-1} \alpha^{-1} y \alpha$, commuting with $\alpha$ and $y$, we have

$$
\begin{aligned}
\mathbf{1}=\beta \beta^{-1} & =\alpha^{-1} y \alpha^{2} y\left(\beta^{-1}\right) \alpha^{-1} y^{-2} \\
& =\alpha^{-1} y \alpha^{2} y\left(y^{-1} \alpha^{-1} y \alpha\right) \alpha^{-1} y^{-2} \\
& =\alpha^{-1} y \alpha y^{-1}
\end{aligned}
$$

By taking the inverse, we get $1=y \alpha^{-1} y^{-1} \alpha$, and thus $y^{-1} \alpha y=\alpha$. But $y^{-1} \alpha y=\alpha \beta$, so these two equations imply that $\beta=1$, which is impossible since $\beta$ is the meridian of the fibre $T^{2}-D^{2}$ in $\Gamma$, which is not capped off by the process. Hence, $H$ cannot be the subgroup $f_{*}\left(\pi_{1}(S)\right)$ in $\pi_{1}(\Gamma)$ when $S$ is the punctured torus. This completes the proof of Theorem 3.4 .

Actually the argument of Case 1 can be modified for the punctured Moebius band case, also. However, we are not very much concerned with this case since the punctured Moebius band would be one-sided.

\section{Separability of closed surfaces in $\Gamma$}

A natural question to ask next is the following: does every incompressible immersion of a surface in $\Gamma$ lift to an embedding in some finite cover of $\Gamma$ ? We begin with the following definition.

Definition 4.1: Let $f: S \rightarrow M$ be a proper, $\pi_{1}$-injective immersion of a connected surface $S$ into a 3-manifold $M . S$ is called separable if there is a finite cover $\widehat{M}$ of $M$ such that $f$ lifts to $\widehat{f}: S \rightarrow \widehat{M}$ as an embedding.

We shall assume that all such immersions are least-area maps in their homotopy classes. Let us first consider closed surfaces. We begin with the following, somewhat surprising lemma.

LEMMA 4.2. Suppose $f: S \rightarrow \Gamma$ is a $\pi_{1}$-injective proper immersion of a connected closed orientable surface into $\Gamma$, and $S$ is not $S^{2}$. Then, $S$ must be the torus.

Proof: We use the graph-manifold description of $\Gamma$ (Lemma 2.1 and Figure 2). Cut open $\Gamma$ along the glued torus $T$ to get $\Gamma^{\prime}=P \times S^{1}$, where $P$ is the pair of pants. Define $f^{\prime}: S^{\prime} \rightarrow \Gamma^{\prime}$, where $S^{\prime}=S-f^{-1}(T)$, possibly disconnected. Each component of $f^{\prime}\left(S^{\prime}\right)$ is $\pi_{1}$-injective (by least area) in $\Gamma^{\prime}$, so assume it is either horizontal or vertical in $\Gamma^{\prime}$. But if a component is horizontal, it must be transverse to every fibre of $\Gamma^{\prime}$, meeting every component of $\partial \Gamma^{\prime}$. This contradicts the hypothesis that $S$ is a proper immersion of a closed surface. Hence, each component is vertical. This implies that $f(S)$ is disjoint from $T$ since the gluing does not preserve the vertical fibres. Hence, $S=S^{\prime}$ and is immersed in $\Gamma^{\prime}$, and it is a union of Seifert fibres; therefore, it must be the torus. 
This lemma means that $f(S)$ is a product of some closed (but not necessarily simple) curve $c$ on the fibre $P$ with an $n$-degree covering of $S^{1}$. (c represents the meridian of $S$ ). We now state one of the main theorems about surfaces in $\Gamma$.

Theorem 4.3. Suppose $f: S \rightarrow \Gamma$ is a $\pi_{1}$-injective proper immersion of an orientable closed connected surface $S$ into $\Gamma$. Then, $S$ is separable.

Proof: The result is trivial if $S=S^{2}$, so by Lemma 4.2, we may suppose that $S$ is the torus. This time, use the HNN-extension model of $\Gamma$ (Theorem 2.3 and Figure 5). Denote $T \times\{0\}$ by $T_{0}$ and $T \times\{1\}$ by $T_{1}$. With this description (Figure 3 and Figure 4), we use the term "connecting annulus" $A$ to refer to the thickened annulus $A \times I$ connecting the 2-dimensional neighbourhoods $N_{0}$ of $\mu$ in $T_{0}$ and $N_{1}$ of $\lambda$ in $T_{1}$. Let us refer to $T \times I$ as $\Gamma^{\prime}$. This is obtained after $\Gamma$ is cut along the middle of the connecting annulus $A$. We have the following two cases.

CASE 1. $S \cap A=\varnothing$. Here, $S \subset \Gamma^{\prime}$. In this case, $S$ can be homotoped to cover the torus $T \times\{1 / 2\}$ in $\Gamma^{\prime}$. Let $\rho: S \rightarrow T \times\{1 / 2\}$ be this covering. $\rho_{*}\left(\pi_{1}(S)\right)$ is some $\mathbb{Z} \oplus \mathbb{Z}$ subgroup in $\pi_{1}(T)=\mathbb{Z} \oplus \mathbb{Z}$. Then, since $\mathbb{Z} \oplus \mathbb{Z}$ is LERF, there exists a finite cover $\widehat{\Gamma^{\prime}}$ of $\Gamma^{\prime}=T \times I$ in which $\rho$ lifts to $\widehat{\rho}: S \rightarrow \widehat{\Gamma}^{\prime}$ as an embedding. Let $\partial \widehat{\Gamma^{\prime}}=T_{0}^{\prime} \sqcup T_{1}^{\prime}$. There are some $m_{0}$ and $l_{0}$, positive integers, such that on $T_{0}^{\prime}$ we see $m_{0}$ copies of the meridian curves $\mu_{1}^{\prime}, \mu_{2}^{\prime}, \ldots, \mu_{m_{0}}^{\prime}$, each covering the original meridian $\mu \subset T_{0}$ with some degree $l_{0}$. On $T_{1}$, then, there are $l_{1}$ copies of the longitude curves $\lambda_{1}^{\prime}, \lambda_{2}^{\prime}, \ldots, \lambda_{l_{1}}^{\prime}$, each covering the original $\lambda \subset T_{1}$ with degree $m_{1}$ (and we have $m_{1} l_{1}=m_{0} l_{0}$ ). Next, we create the "dual space" $\widehat{\Gamma^{*}}$, a covering space of $\Gamma^{\prime}$ just like $\widehat{\Gamma^{\prime}}$ except the meridian and the longitude are reversed. Let $\partial \widehat{\Gamma^{*}}=T_{0}^{*} \cup T_{1}^{*}$. As before, $T_{0}^{*}$ has $l_{1}$ copies of the meridian curves $\mu_{1}^{*}, \ldots, \mu_{l_{1}}^{*}$, each covering $\mu$ with degree $m_{1}$, and $T_{1}^{*}$ has $m_{0}$ copies of the longitude curves $\lambda_{1}^{*}, \ldots, \lambda_{m_{0}}^{*}$, each covering $\lambda$ with degree $l_{0}$. These degrees on $\partial \widehat{\Gamma^{\prime}}$ and $\partial \widehat{\Gamma^{*}}$ match up, so we can join the $\mu_{i}^{\prime}$ with the $\lambda_{i}^{*}\left(m_{0}\right.$ of these), and the $\lambda_{i}^{\prime}$ with the $\mu_{i}^{*}$ ( $l_{1}$ of these). The result, $\widehat{\Gamma}$, is a finite covering space for $\Gamma$ in which $S$ is embedded.

This procedure will be used in Case 2 as well. In fact, it is highly useful to describe this process using graphs with edges and half-edges. $\hat{\Gamma}^{\prime}$ can be represented by a finite graph with one vertex $v$ (for $\widehat{\Gamma}^{\prime}$ ), $m_{0}$ incoming half-edges labelled $l_{0}$ (for $\left.\mu_{1}^{\prime}, \mu_{2}^{\prime}, \ldots, \mu_{m_{0}}^{\prime}\right)$, and $l_{1}$ outgoing half-edges labelled $m_{1}$ (for $\lambda_{1}^{\prime}, \ldots \lambda_{l_{1}}^{\prime}$ ). Note that the incoming half-edges represent the curves on $\partial \widehat{\Gamma}^{\prime}$ covering the meridian of $\partial \Gamma^{\prime}$ with the labelled degrees, and the outgoing half-edges represent the curves on $\partial \widehat{\Gamma^{\prime}}$ covering the longitude of $\partial \Gamma^{\prime}$ with the labelled degrees. In this way, each covering space $\widehat{\Gamma}^{\prime}$ of $\Gamma^{\prime}$ can be represented by a vertex with an appropriate number of labelled, directed half-edges (see Figure 8 for $\hat{\Gamma}^{\prime}$ when $m_{0}=m_{1}=3, l_{0}=l_{1}=2$ ).

Note that the dual space $\widehat{\Gamma^{*}}$ has the graph representation where all the edges are 

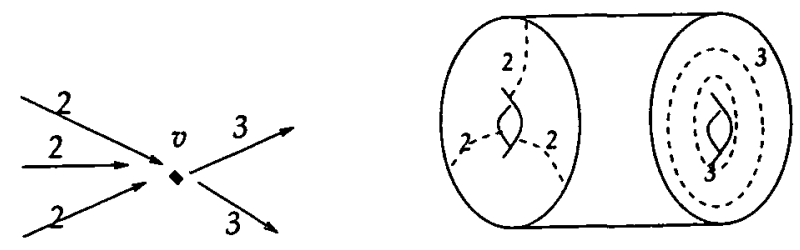

Figure 8. $\widehat{\Gamma}^{\prime}$ when $m_{0}=m_{1}=3, l_{0}=l_{1}=2$

reversed; therefore, we can connect the half-edges to "close up" the space (see Figure 9 ) and obtain a covering space $\widehat{\Gamma}$ of $\Gamma$. Note that the surface $S$ is embedded entirely in $\widehat{\Gamma}^{\prime}$, and the sole purpose for constructing $\widehat{\Gamma^{*}}$ is to close up the space $\widehat{\Gamma^{\prime}}$ so that $\widehat{\Gamma}$ becomes a covering space of $\Gamma$. The covering projection $\widehat{\Gamma} \rightarrow \Gamma$ is an extension of the covering $\widehat{\Gamma}^{\prime} \rightarrow \Gamma^{\prime}$; the covering $\widehat{\Gamma^{*}} \rightarrow \Gamma^{\prime}$ is defined naturally as the dual of $\widehat{\Gamma}^{\prime}$, and the degree- $m_{i}$ (and $-l_{i}$ ) maps of the annuli joining $\widehat{\Gamma^{\prime}}$ and $\widehat{\Gamma^{*}}$ cover the connecting annuli $A_{i}$ (see Figure 4), making the map $\widehat{\Gamma} \rightarrow \Gamma$ a covering projection.

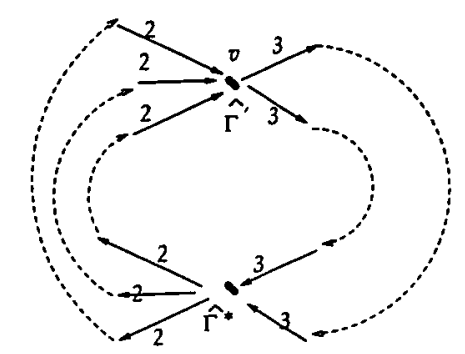

Figure 9. Covering Space $\widehat{\Gamma}$ of $\Gamma$

CASE 2. $S \cap A \neq \varnothing$. Assume that $f$ is transverse to $A$. Let $S^{\prime}=S-f^{-1}(A)$, and cut $\Gamma$ open along $A$ to get $f^{\prime}: S^{\prime} \rightarrow \Gamma^{\prime}$. Observe that we can now identify $A$ as $N_{0} \subset T_{0}$ and $N_{1} \subset T_{1}$ as in Section 2. Now, $f^{-1}(A)$ is a 1-manifold on $S$, so by making both $A$ and $f$ least area in their homotopy classes (so none of the circles are trivial), we may assume $f^{-1}(A)$ is a disjoint union of essential circles of the torus $S$. Each component $C$ of $S^{\prime}$ is immersed properly and $\pi_{1}$-injectively in $\Gamma^{\prime}$, and $\partial C \subset A$. All these circles are parallel in the torus $S$, implying that each $C$ is an annulus. Denote the two boundary components of the annulus $C$ by $\partial_{0} C$ and $\partial_{1} C . f\left(\partial_{0} C\right)$ and $f\left(\partial_{1} C\right)$ are in the same component of $\partial \Gamma^{\prime}$ since otherwise $C$ would be an annulus in $\Gamma^{\prime}$ joining $\lambda$ to $\mu$ in $\pi_{1}\left(\Gamma^{\prime}\right)$, which is impossible. Suppose for now that $f\left(\partial_{i} C\right)$ are both homotopic to $\mu^{d} \in \pi_{1}\left(T_{0}\right)$ for some $d$. Again, by least area, $C$ cannot be homotopic rel $\partial C$ into $A=N_{0}$. So $C$ must go around $T_{0}$ at least once in the longitude direction. Hence, $C$ is an annulus "beginning" at $\partial_{0} C$ on $N_{0} \subset T_{0}$, going "around" $T$ (in $T \times I=\Gamma^{\prime}$ ) a 
number of times, and "ending" at $\partial_{1} C$, also on $N_{0} \subset T_{0}$. Clearly, a similar condition holds if the boundary curves of $C$ are in $N_{1} \subset T_{1}$. In fact, as $S$ is a torus, these must match together to close up. So $S^{\prime}=S \backslash f^{-1}(A)$ consists of an even number of annuli, where the annuli have their boundary circles in $T_{0}$ and $T_{1}$ alternatingly. Let us refer to those annuli with boundary in $N_{0}$ ("the left-hand side" of $A$ ) as the "meridian annuli," and the other ones on $N_{1}$ as the "longitude annuli." We can then write $S^{\prime}=\bigcup C_{i}$, where an odd index indicates a meridian annulus and an even index indicates a longitude annulus.

We can describe each component $C_{i}$ of $S^{\prime}$ by a pair $\left(m_{i}, l_{i}\right)$ as follows. Suppose $C_{i}$ is a meridian annulus. Then, $m_{i}$ is the integer such that each component of $f\left(\partial C_{i}\right)$ covers the meridian of the fibre torus $T$ of $\Gamma^{\prime}$ by the degree- $m_{i}$ cover, and $l_{i}$ indicates how many times the $I$-factor of $C_{i} \cong S^{1} \times I$ goes around the longitude of $T$. If $C_{i}$ is a longitude annulus, one can similarly describe it by $\left(m_{i}, l_{i}\right)$; each component of $f\left(\partial C_{i}\right)$ covers the longitude of $T$ by the degree- $l_{i}$ cover, and the $I$-factor of $C_{i}$ goes around the meridian $m_{i}$ times.

We now return to the construction of a finite-degree cover $\widehat{\Gamma}$ of $\Gamma$. The procedure is similar to that of Case 1. Say there are $2 k$ components of $S \backslash f^{-1}(A)$. Since $C_{1}, C_{3}, \ldots$ are the meridian annuli while $C_{2}, C_{4}, \ldots$ are the longitude ones, we must have $m_{1}=$ $l_{2}=m_{3}=l_{4}=\cdots=l_{2 k}$. Let this number be $d \in \mathbb{Z}^{+}$(for "degree").

Now, define the $(x, y)$-cover of $T$ to mean the covering torus $T^{\prime}$ defined by the map $\mathbb{Z} \oplus \mathbb{Z} \rightarrow \mathbb{Z}_{x} \oplus \mathbb{Z}_{y}$, sending each generator to the corresponding generator. For $C_{i}$, described by $\left(m_{i}, l_{i}\right)$, where $i$ is odd, take $\Gamma_{i}^{\prime}$ to be $T_{i}^{\prime} \times I$, where $T_{i}^{\prime}$ is the $\left(d, l_{i}+1\right)$ cover of $T$. We use $l_{i}+1$ so that we can be sure that $C_{i}$ is embedded in $\Gamma_{i}^{\prime}$. $C_{i}$ enters $\Gamma_{i}^{\prime}$ at one component of the pre-image of $\mu$ and leaves $\Gamma_{i}^{\prime}$ at another, which will later enable us to join the embedded $C_{i}$ together. For $C_{i}, i$ even, let $\Gamma_{i}^{\prime}=T_{i}^{\prime} \times I$, where $T_{i}^{\prime}$ is the $\left(m_{i}+1, d\right)$-cover of $T$. Again, $C_{i}$ embeds in $\Gamma_{i}^{\prime}$. We obtain $2 k$ distinct covering spaces of $\Gamma^{\prime}$, possibly of different degrees. But the embedded $C_{i}$ can be connected by appropriate covers $A_{i}$ of $A$ because $d=m_{1}=l_{2}=\cdots=l_{2 k}$. So, the $2 k$ covering spaces $\Gamma_{i}^{\prime}$ of $\Gamma^{\prime}$, joined together by the $A_{i}$, contain an embedded copy of $S$.

We now need to make this object into a covering space for $\Gamma$ by "closing up" those pre-images of $N_{0}$ and $N_{1}$. Again, we reverse the longitude and the meridian of $\Gamma_{i}^{\prime}$ to create the covering spaces $\Gamma_{i}^{*}$ of $\Gamma^{\prime}$, dual to $\Gamma_{i}^{\prime}$. Now, $\bigcup_{i=1}^{2 k} \Gamma_{i}^{\prime}$ can be represented by the graph seen in Figure 10, where the vertex $v_{i}$ represents $\Gamma_{i}^{\prime}$, the cover of $\Gamma^{\prime}$ having the right number of incoming and outgoing edges of appropriate degrees and two of the edges are connected to the adjacent vertices $v_{i-1}$ and $v_{i+1}$ just as the $A_{i}$ connect the $C_{i}$. The fact that the $A_{i}$ join the $C_{i}$ to complete the embedded torus $S$ is reflected by the fact that there is a complete circle connecting the $v_{i}$ by edges labelled $d$. We 

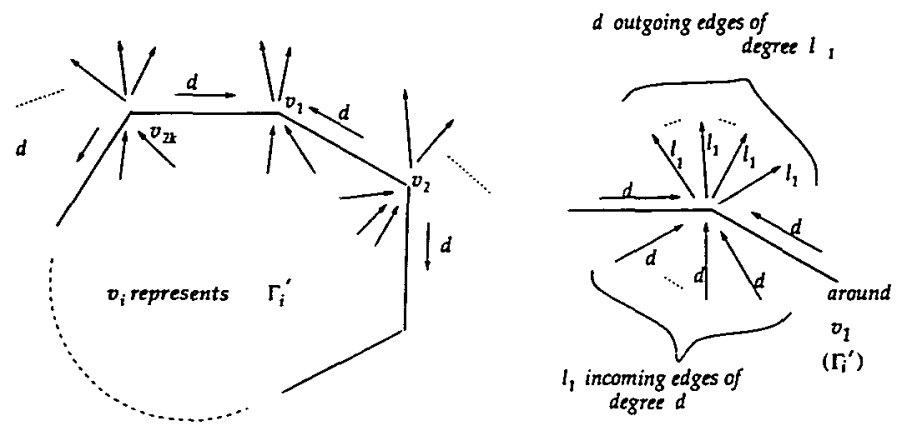

Figure 10. Space containing $S$ as an embedded surface

may have many half-edges now. But once again, the dual spaces $\Gamma_{i}^{*}$ are represented by the same graph except that the orientations of the edges are reversed. Join the $\Gamma_{i}^{*}(1 \leqslant i \leqslant 2 k)$ exactly as the $\Gamma_{i}^{\prime}$ so that the $\Gamma_{i}^{*}$ also makes a circle. We can now pair up the "open" half-edges of $\Gamma_{i}^{\prime}$ with those of $\Gamma_{i}^{*}$ to create $\widehat{\Gamma}$, a covering space of $\Gamma$, since the degrees, orientations, and the number of the half-edges match up by construction. $S$ is embedded in $U \Gamma_{i}^{\prime} \subset \widehat{\Gamma}$, so $\widehat{\Gamma}$ is a desired finite cover of $\Gamma$. Incidentally, the degree of $\widehat{\Gamma}$ over $\Gamma$ is $2 d\left(2 k+\sum_{i \text { odd }}^{2 k} l_{i}+\sum_{i \text { even }}^{2 k} m_{i}\right)$.

\section{Separability OF NON-CLOSED SURFACES IN $\Gamma$}

The next question is for properly immersed incompressible surfaces with boundary. If $f: S \rightarrow M$ is a proper immersion of a compact orientable surface into a graph manifold $M=\bigcup M_{i}$, we say $S$ is horizontal if $f(S) \cap M_{i}$ is horizontal or empty for all $i$. Of course, many immersed surfaces are neither horizontal nor vertical, but we can assume that each non-empty intersection $f(S) \cap M_{i}$ is either horizontal or vertical (up to homotopy) by the theory of least-area maps (see Hass and Scott [6] and Freedman, Hass, and Scott [4]). We now need one more definition to state the crucial criterion proved by Rubinstein and Wang [12].

Definition 5.1: Suppose $f: S \rightarrow M$ is a $\pi_{1}$-injective horizontal proper immersion into a graph manifold $M$, where $M=\bigcup M_{i}$, glued along a family $\mathcal{T}$ of tori. Assume transversality so that $f^{-1}(T)$ is a family $\mathcal{C}$ of disjoint simple closed curves $c$ on $S$. Let $\gamma$ be an oriented simple closed curve on $S$, parameterised by $t \in[0,1]$. As $t$ increases, $\gamma$ can meet $f^{-1}(\mathcal{T})$ at $c \in \mathcal{C}$. Say at $c, f(\gamma)$ goes from $M_{i}$ to $M_{j}$, where $f_{i}(c)=f(c)$ on $\partial M_{i}$ and $f_{j}(c)=f(c)$ on $\partial M_{j}$. Then, define

$$
\rho_{c}=\frac{\left|t_{i} \cap f_{i}(c)\right|}{\left|t_{j} \cap f_{j}(c)\right|}
$$


where $t_{i}$ (and $t_{j}$ ) is a regular fibre of $M_{i}$ (and of $M_{j}$ ). If $\gamma$ intersects $\mathcal{C}$ at $c_{1}, c_{2}, \ldots, c_{k}$ in that order, then define

$$
s_{\gamma}=\prod_{i=1}^{k} \rho_{c_{i}}
$$

Hence, for any $c \in \mathcal{C}$ intersecting $\gamma, \rho_{c}$ is a ratio: the numerator measures how many times $f_{i}(c)$ intersects a regular fibre of $\partial M_{i}$ and the denominator similarly measures how many times $f_{j}(c)$ intersects a regular fibre of $\partial M_{j}$, the "other side." For $\gamma$ not intersecting $\mathcal{C}$, define $s_{\gamma}=1$. For every oriented simple closed curve $\gamma \subset S$, then, $s_{\gamma}$ is well-defined.

As $\gamma$ goes from $M_{i}$ to $M_{j}$ at $c \in \mathcal{C}, f_{i}(c)$ is often represented (up to homotopy) by a pair of coordinates $(\alpha, \beta)$ on $\partial M_{i}$. The corresponding coordinates $\left(\alpha^{\prime}, \beta^{\prime}\right)$ for $f_{j}(c)$ on $\partial M_{j}$ can be obtained by

$$
\left(\begin{array}{l}
\alpha^{\prime} \\
\beta^{\prime}
\end{array}\right)=\left(\begin{array}{ll}
h_{11} & h_{12} \\
h_{21} & h_{22}
\end{array}\right)\left(\begin{array}{l}
\alpha \\
\beta
\end{array}\right),
$$

where the $2 \times 2$ matrix represents the gluing. If we use the convention that the first coordinate is the meridian (horizontal), then $\left|t_{i} \cap f_{i}(c)\right|=\alpha$, and $\left|t_{j} \cap f_{j}(c)\right|=\alpha^{\prime}$, making $\rho_{c}=\alpha / \alpha^{\prime}$. Then, we can write

$$
s_{\gamma}=\prod_{i=1}^{k} \frac{\alpha_{i}}{\alpha_{i}^{\prime}}
$$

and the $\beta_{i}$ and $\beta_{i}^{\prime}$ are irrelevant.

Now, we are ready to state Rubinstein and Wang's criterion [12]:

THEOREM 5.2. Suppose $f: S \rightarrow M$ is a $\pi_{1}$-injective proper horizontal immersion of a compact connected surface $S$ into a graph manifold $M$. Then, $S$ is separable if and only if $s_{\gamma}=1$ for each oriented simple closed curve $\gamma \subset S$.

We shall soon use this criterion to construct a surfaces not separable in $\Gamma$; however, the construction requires some more definitions.

Definition 5.3: Let $f: S \rightarrow M$ be a proper map of a connected surface (not $D^{2}$ ) into some 3-manifold. $S$ is called arc-incompressible if the following holds: suppose there is a disk $D$ in $M$ with the boundary consisting of a proper arc $\delta$ in $f(S)$ and an arc $\gamma$ in $\partial M$ sharing the same endpoints such that $D \cap f(S)=\delta$. Then, there is a path $\delta^{\prime}$ in $f(\partial S) \subset \partial M$ such that $\delta \cup \delta^{\prime}$ bounds a disc in $S$.

If $S$ is not arc-incompressible, $S$ is said to be arc-compressible, and the disc $D$ such that $\partial D=\delta \cup \gamma$ is called a compressing disc.

We shall use this to prove the following. 
Lemma 5.4. Suppose $M=\bigcup_{T} M_{i}$ is a graph manifold. Let $f: S \rightarrow M$ be a proper immersion of a connected compact surface. If each component of $S-f^{-1}(\mathcal{T})$ is a $\pi_{1}$-injective horizontal surface in the corresponding $M_{i}$, then $S$ is also a $\pi_{1}$-injective horizontal surface in $M$.

Proof: By definition, $S$ is horizontal. Suppose $S$ fails to be $\pi_{1}$-injective in $M$. Then, there is a compressing disc $D$ in $M$. But $D$ cannot be disjoint from all the tori in $\mathcal{T}$ since that would make a component of $S \backslash f^{-1}(\mathcal{T})$ compressible in $M_{i}$, contrary to the hypothesis. So the compressing disc $D$ intersects some torus $T \in \mathcal{T}$. In $D, D \cap(U \mathcal{T})$ is a disjoint collection of proper arcs and circles. But by least area of the map and the tori in $\mathcal{T}$, there are no circles and that any outermost arc represents a compressing disc $D_{i}$. Now, a horizontal surface $C_{i}$ lifts to an embedding $e_{i}: C_{i} \rightarrow C_{i} \times S^{1}=\widehat{M}_{i}$ in a finite cover $\widehat{M}_{i}$ of $M_{i}$, and $D_{i}$ also lifts to a disc $\widehat{D_{i}}$ in $C_{i} \times S^{1}$. But this implies that $C_{i}$ is arc-compressible in $C_{i} \times S^{1}$, which is absurd.

To give an explicit description of a surface which is not separable in $\Gamma$, we shall need the following construction. Let $M=F \times S^{1}$, where $F$ is some compact surface. Suppose $f: S \rightarrow M$ is a proper immersion such that $f(S) \subset F \times\{0\}$ ("really horizontal"), and let $\gamma \subset f(S)$ be a simple closed curve or a simple proper arc in $f(S)$. Then, by the Dehn twist along $\gamma$ of degree $d(d \in \mathbb{Z})$, we mean the following modification of $f$ to obtain $f^{\prime}: S \rightarrow M$.

Take a regular neighbourhood $\gamma \times I$ of $\gamma$, with two boundary components $\gamma_{0}$ and $\gamma_{1}$. Fix a point $x$ of $\gamma$, and let $\delta_{x}$ be the path $x \times I$ from $(x, 0)$ to $(x, 1)$ in $F \times\{0\}$. Now, replace $\delta_{x}$ with $\delta_{x}^{\prime}$, the path from $(x, 0)$, traveling in the $S^{1}$-direction $d$ times and terminating at $(x, 1)$. This is also referred to as a " $d$-floor staircase construction" for an obvious reason. Now, replace $\gamma \times I \subset F \times\{0\}$ with $\bigcup_{x \in \gamma} \delta_{x}^{\prime}$ (homeomorphic to $\gamma \times I$ ), and take this new image to be $f^{\prime}(S)$. This resulting map $f^{\prime}$ is still horizontal because the image of the immersed surface is still transverse to the fibres. Being horizontal, $f^{\prime}$ is still $\pi_{1}$-injective.

THEOREM 5.5. There is a proper immersion $f: S \rightarrow \Gamma, \pi_{1}$-injective, where $S$ is a connected orientable surface and is not separable.

Proof: $S=S_{1} \cup S_{2}$ is constructed as follows. Let $P$ denote the pair of pants described as a disk with two holes labelled $T_{x}$ and $T_{y}$, corresponding to the generators $x$ and $y$ of $\pi_{1}(P)$. Let $S_{1}$ be the double cover of $P$ defined by the homomorphism $\Theta: \pi_{1}(P)=\langle x, y\rangle \rightarrow \mathbb{Z}_{2}$ sending $x \longmapsto 0$ and $y \longmapsto 1$. This defines $p_{1}: S_{1} \rightarrow P$ such that $p_{1}^{-1}(x)$ is two disjoint circles each covering $x$ by degree 1 , and $p_{1}^{-1}(y)$ is one circle covering $y$ by degree 2 . This map $p_{1}$ describes a really horizontal surface in $\Gamma^{\prime}=P \times S^{1}$ (as described in Figure 2) so these pre-image circles have coordinates $(1,0),(1,0)$ for $p_{1}^{-1}(x)$ and $(2,0)$ for $p_{1}^{-1}(y)$. Now, do the Dehn twist of degree 1 as shown (Figure 
11) along two paths of $S_{1}$. Now the coordinates are $(1,0),(1,1)$, and $(2,1)$, where the first one is the circle left untouched in Figure 11. For $S_{2}$, reverse $x$ and $y$. Define $p_{2}: S_{2} \rightarrow P$ by $\Theta^{\prime}: \pi_{1}(P) \rightarrow \mathbb{Z}_{2}$ sending $x \longmapsto 1$, and $y \longmapsto 0$. Do the Dehn twist as shown (Figure 12) of degree -1 to get the coordinates $(2,-1)$ for $p_{2}^{-1}(x)$ and $(1,0)$ and $(1,-1)$ for $p_{2}^{-1}(y)$. Now, in $P \times S^{1}$, we see two surfaces $S_{i}, i=1,2$ with coordinates shown (Figure 13).

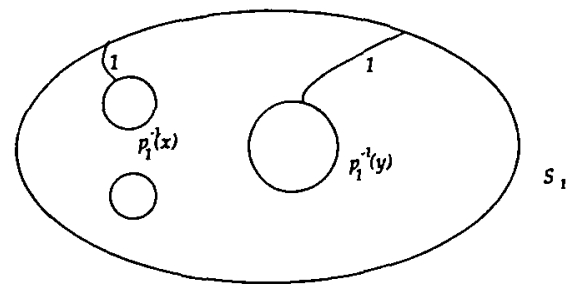

Figure 11. $S_{1}$ in the construction

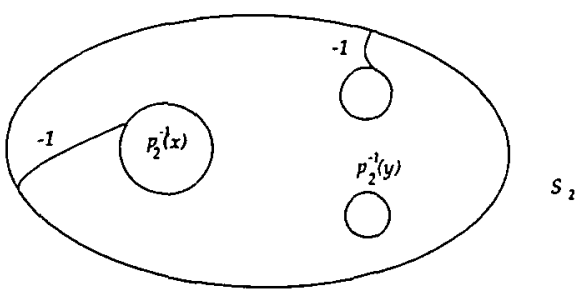

Figure 12. $S_{2}$ in the construction

Immerse $S=S_{1} \cup S_{2}$ such that the circle $(1,1)$ is glued (correctly) to $(2,1)$ by $h=\left(\begin{array}{ll}1 & 1 \\ 0 & 1\end{array}\right),(1,0)$ is glued to $(1,0)$, and $(2,-1)$ is glued to $(1,-1)$. Note that this surface is the connected sum of two punctured tori properly immersed in $\Gamma$. This immersion is $\pi_{1}$-injective by Lemma 5.2 above.

Consider $\gamma$ shown in Figure 13. $s_{\gamma}=1 / 2$ (or 2, depending on the orientation of $\gamma$ ). By Rubinstein and Wang's criterion (Theorem 5.2), $S$ is not separable.

It is clear that the criterion gives many other horizontal surfaces that are not separable in $\Gamma$. All one needs to do is to construct surfaces similar to the one in Theorem 5.5 with a loop $\gamma$ such that $s_{\gamma} \neq 1$. This suggests that graph manifolds have many immersed surfaces that are not separable in them.

\section{REFERENCES}

[1] R.B.J.T. Allenby and R.J. Gregorac, 'On locally extended residually finite groups', in 


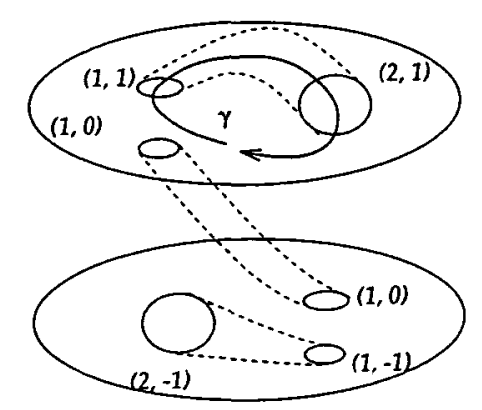

Figure 13. Non-separable surface in $\Gamma$

Conference on group theory, Lecture Notes in Mathematics 319 (Springer-Verlag, Berlin, Heidelberg, New York, 1973), pp. 9-17.

[2] R.G. Burns, 'A note on free groups', Proc. Amer. Math. Soc. 23 (1969), 14-17.

[3] R.G. Burns, A. Karrass and D. Solitar, 'A note on groups with separable finitely generated subgroups', Bull. Austral. Math. Soc. 36 (1987), 153-160.

[4] M. Freedman, J. Hass and P. Scott, 'Least area incompressible surfaces in 3-Manifolds', Invent. Math. 71 (1983), 609-642.

[5] M. Hall, 'Coset representations in free groups', Trans. Amer. Math. Soc. 67 (1949), 421-432.

[6] J. Hass and P. Scott, 'The existence of least area surfaces in 3-Manifolds', Trans. Amer. Math. Soc. 310 (1988), 87-114.

[7] J. Hempel, 'Residual finiteness of surface groups', Proc. Amer. Math. Soc. 32 (1972), 323.

[8] A. Karrass and D. Solitar, 'On finitely generated subgroups of a free group', Proc. Amer. Math. Soc. 22 (1969), 209-213.

[9] D. Long and G.A. Niblo, 'Subgroup separability and 3-manifold groups', Math. Z. 207 (1991), 209-215.

[10] R. Lyndon and P. Schupp, Combinatorial group theory (Springer-Verlag, Berlin, Heidelberg, New York, 1977).

[11] W. Magnus, 'Residually finite groups', Bull. Amer. Math. Soc. 75 (1969), 305-315.

[12] J.H. Rubinstein and $S$. Wang, 'On $\pi_{1}$-injective surfaces in graph manifolds', (preprint).

[13] P. Scott, 'Subgroups of surface groups are almost geometric', J. London Math. Soc. (2) 17 (1978), 555-565.

[14] P. Scott, 'The geometries of 3-manifolds', Bull. London Math. Soc. 15 (1983), 401-487.

Department of Mathematics

University of Melbourne

Parkville Vic 3052

Australia

e-mail: saburo@maths.mu.oz.au 